\title{
LATTICE STABILITY FOR ATOMISTIC CHAINS MODELED BY LOCAL APPROXIMATIONS OF THE EMBEDDED ATOM METHOD
}

\author{
XINGJIE HELEN LI AND MITCHELL LUSKIN
}

\begin{abstract}
The accurate approximation of critical strains for lattice instability is a key criterion for predictive computational modeling of materials. In this paper, we present a comparison of the lattice stability for atomistic chains modeled by the embedded atom method (EAM) with their approximation by local Cauchy-Born models. We find that both the volume-based local model and the reconstruction-based local model can give $\mathrm{O}(1)$ errors for the critical strain since the embedding energy density is generally strictly convex. The critical strain predicted by the volumebased model is always larger than that predicted by the atomistic model, but the critical strain for reconstruction-based models can be either larger or smaller than that predicted by the atomistic model.
\end{abstract}

\section{INTRODUCTION}

Predictive multiscale computational methods must be accurate near lattice instabilities that characterize the formation and movement of cracks, dislocations, and grain boundaries. In this paper, we present analytic results comparing the lattice instabilities predicted by an atomistic chain modeled by the embedded atom method (EAM) with the lattice instabilities predicted by local approximations of the atomistic model.

Since it is not possible to compute large enough fully atomistic systems to accurately approximate the interaction of local defects with long-range elastic fields, atomistic-to-continuum coupling methods have been proposed [1,2, 5, 11 13, 16, 18, 20]. For crystalline solids, the continuum region is generally computed by coarse-graining a local approximation of the nonlocal atomistic model. An atom in the nonlocal atomistic region interacts with all of its neighbors within a cutoff radius. In the continuum region, the Cauchy-Born rule is used to derive a local model that approximates the interactions of atoms beyond their nearest neighbors by modified interactions of their nearest neighbors.

To verify that an atomistic-to-continuum coupling method accurately reproduces the lattice stability of the fully atomistic model, it is necessary to first verify that the local (continuum) model itself reproduces the lattice stability of the fully atomistic model. Even if the local model reproduces the lattice stability of the fully atomistic model, the atomistic-to-continuum coupling method may not reproduce the lattice stability of the fully atomistic model because of the error introduced by the coupling [3, 19].

It has been proven in [3] that the Cauchy-Born local model reproduces the lattice stability for an atomistic chain modeled by Lennard-Jones type pair interaction (we note that the volume-based

Date: June 20, 2018.

2000 Mathematics Subject Classification. 65Z05,70C20.

Key words and phrases. quasicontinuum, error analysis, atomistic to continuum, embedded atom model, quasinonlocal.

This work was supported in part by DMS-0757355, DMS-0811039, the Institute for Mathematics and Its Applications, and the University of Minnesota Supercomputing Institute. This work was also supported by the Department of Energy under Award Number de-sc0002085. 
and reconstruction-based local models are equal for a pair potential interaction). And it has been proven for multidimensional lattices that the set of stable uniform strains for the atomistic model is a subset of the set of uniform strains for the Cauchy-Born volume-based local model [6, 8, but the equality of these sets has not been demonstrated analytically. As a matter of fact, numerical experiments in [8] suggest that the the inclusion is strict in some cases.

In this paper, we prove for an atomistic chain that not only are the sets of stable uniform strains different for the atomistic model and the local models for a many-body potential, but the set of stable uniform strains can be different for volume-based and reconstruction-based local models. We will focus our analysis on the embedded atom method [7, 9, 14], which is an empirical many-body potential that is widely used to model FCC metals such as copper and aluminum. We identify the critical assumptions for the pair potential, electron density function, and embedding function to study the lattice stability of the atomistic and the different local models. We find that both the volume-based local model and the reconstruction-based local model can give $\mathrm{O}(1)$ errors for the critical strain since the embedding energy density is generally strictly convex.

In Section 2, we present the notation used in this paper. We define the displacement space $\mathcal{U}$ and the deformation space $\mathcal{Y}_{F}$. We then introduce the norms we will use to estimate the modeling error and the displacement gradient error. In Section 3, we briefly review the formulae of the fully atomistic EAM model and the volume-based and the reconstruction-based local quasicontinuum (QCL) model, respectively.

In Section 4, we give precise stability estimates for the fully atomistic model, the volume-based and the reconstruction-based local models for a uniformly strained chain. We then compare the stability conditions of each model under different assumptions. We summarize our results and discuss extensions to multidimensional issues in the Conclusion.

\section{Notation}

In this section, we present the notation used in this paper. We define the scaled reference lattice

$$
\epsilon \mathbb{Z}:=\{\epsilon \ell: \ell \in \mathbb{Z}\},
$$

where $\epsilon>0$ scales the reference atomic spacing and $\mathbb{Z}$ is the set of integers. We then deform the reference lattice $\epsilon \mathbb{Z}$ uniformly into the lattice

$$
F \in \mathbb{Z}:=\{F \in \ell: \ell \in \mathbb{Z}\}
$$

where $F>0$ is the macroscopic deformation gradient, and we define the corresponding deformation $\mathbf{y}_{F}$ by

$$
\left(\mathbf{y}_{F}\right)_{\ell}:=F \epsilon \ell \text { for }-\infty<\ell<\infty .
$$

For simplicity, we consider the space $\mathcal{U}$ of $2 N$-periodic zero mean displacements $\mathbf{u}=\left(u_{\ell}\right)_{\ell \in \mathbb{Z}}$ from $\mathbf{y}_{F}$ given by

$$
\mathcal{U}:=\left\{\mathbf{u}: u_{\ell+2 N}=u_{\ell} \text { for } \ell \in \mathbb{Z}, \text { and } \sum_{\ell=-N+1}^{N} u_{\ell}=0\right\},
$$

and we thus admit deformations $\mathbf{y}$ from the space

$$
\mathcal{Y}_{F}:=\left\{\mathbf{y}: \mathbf{y}=\mathbf{y}_{F}+\mathbf{u} \text { for some } \mathbf{u} \in \mathcal{U}\right\}
$$

We set $\epsilon=1 / N$ throughout so that the reference length of the periodic domain is fixed.

We define the discrete differentiation operator, $D \mathbf{u}$, on periodic displacements by

$$
(D \mathbf{u})_{\ell}:=\frac{u_{\ell}-u_{\ell-1}}{\epsilon}, \quad-\infty<\ell<\infty .
$$


We note that $(D \mathbf{u})_{\ell}$ is also $2 N$-periodic in $\ell$ and satisfies the zero mean condition. We will denote $(D \mathbf{u})_{\ell}$ by $D u_{\ell}$. We then define

$$
\left(D^{(2)} \mathbf{u}\right)_{\ell}:=\frac{D u_{\ell}-D u_{\ell-1}}{\epsilon}, \quad-\infty<\ell<\infty,
$$

and we define $\left(D^{(3)} \mathbf{u}\right)_{\ell}$ and $\left(D^{(4)} \mathbf{u}\right)_{\ell}$ in a similar way. To make the formulas concise and more readable, we sometimes denote $D u_{\ell}$ by $u_{\ell}^{\prime}, D^{(2)} u_{\ell}$ by $u_{\ell}^{\prime \prime}$, etc., when there is no confusion in the expressions.

For a displacement $\mathbf{u} \in \mathcal{U}$ and its discrete derivatives, we define the discrete $\ell_{\epsilon}^{2}$ norms by

$$
\|\mathbf{u}\|_{\ell_{\epsilon}^{2}}:=\left(\epsilon \sum_{\ell=-N+1}^{N}\left|u_{\ell}\right|^{2}\right)^{1 / 2}, \quad\left\|\mathbf{u}^{\prime}\right\|_{\ell_{\epsilon}^{2}}:=\left(\epsilon \sum_{\ell=-N+1}^{N}\left|u_{\ell}^{\prime}\right|^{2}\right)^{1 / 2} \text {, etc. }
$$

Finally, for smooth real-valued functions $\mathcal{E}(\mathbf{y})$ defined for $\mathbf{y} \in \mathcal{Y}_{F}$, we define the first and second derivatives (variations) by

$$
\begin{aligned}
\langle\delta \mathcal{E}(\mathbf{y}), \mathbf{w}\rangle & :=\sum_{\ell=-N+1}^{N} \frac{\partial \mathcal{E}}{\partial y_{\ell}}(\mathbf{y}) w_{\ell} \quad \text { for all } \mathbf{w} \in \mathcal{U}, \\
\left\langle\delta^{2} \mathcal{E}(\mathbf{y}) \mathbf{v}, \mathbf{w}\right\rangle & :=\sum_{\ell, m=-N+1}^{N} \frac{\partial^{2} \mathcal{E}}{\partial y_{\ell} \partial y_{m}}(\mathbf{y}) v_{\ell} w_{m} \quad \text { for all } \mathbf{v}, \mathbf{w} \in \mathcal{U} .
\end{aligned}
$$

\section{The Embedded Atom Model and Its Local Approximations.}

In this section, we will give a short description for the next-nearest neighbor atomistic EAM model and its approximations .

3.1. The Next-Nearest Neighbor Atomistic EAM Model. Given deformations $\mathbf{y} \in \mathcal{Y}_{F}$, the total energy per period of the next-nearest neighbor atomistic EAM model is

$$
\mathcal{E}_{\text {tot }}^{a}(\mathbf{y}):=\mathcal{E}^{a}(\mathbf{y})+\mathcal{F}(\mathbf{y}),
$$

where $\mathcal{E}^{a}(\mathbf{y})$ is the total atomistic energy and $\mathcal{F}(\mathbf{y})$ is the total external potential energy. The total atomistic energy $\mathcal{E}^{a}(\mathbf{y})$ is the sum of the embedding energy, $\hat{\mathcal{E}}^{a}(\mathbf{y})$, and the pair potential energy, $\tilde{\mathcal{E}}^{a}(\mathbf{y})$. The energy expression is

$$
\mathcal{E}^{a}(\mathbf{y}):=\hat{\mathcal{E}}^{a}(\mathbf{y})+\tilde{\mathcal{E}}^{a}(\mathbf{y})=\epsilon \sum_{\ell=-N+1}^{N}\left(\hat{\mathcal{E}}_{\ell}^{a}(\mathbf{y})+\tilde{\mathcal{E}}_{\ell}^{a}(\mathbf{y})\right) .
$$

The embedding energy per atom (per atomistic reference spacing $\epsilon$ ) is defined as $\hat{\mathcal{E}}_{\ell}^{a}(\mathbf{y}):=G\left(\bar{\rho}_{\ell}^{a}(\mathbf{y})\right)$, where $G(\bar{\rho})$ is the embedding energy function and $\bar{\rho}_{\ell}^{a}(\mathbf{y})$ is the total electron density at atom $\ell$ :

$$
\bar{\rho}_{\ell}^{a}(\mathbf{y}):=\rho\left(y_{\ell}^{\prime}\right)+\rho\left(y_{\ell}^{\prime}+y_{\ell-1}^{\prime}\right)+\rho\left(y_{\ell+1}^{\prime}\right)+\rho\left(y_{\ell+1}^{\prime}+y_{\ell+2}^{\prime}\right) .
$$

The function $\rho(r / \epsilon)$ is the electron density contributed by an atom at distance $r$.

The pair potential energy per atom (per atomistic reference spacing $\epsilon$ ) is

$$
\tilde{\mathcal{E}}_{\ell}^{a}(\mathbf{y}):=\frac{1}{2}\left[\phi\left(y_{\ell}^{\prime}\right)+\phi\left(y_{\ell}^{\prime}+y_{\ell-1}^{\prime}\right)+\phi\left(y_{\ell+1}^{\prime}\right)+\phi\left(y_{\ell+1}^{\prime}+y_{\ell+2}^{\prime}\right)\right]
$$


where $\phi(r / \epsilon)$ is the pair potential interaction energy [7. Our formulation allows general nonlinear external potential energies $\mathcal{F}(\mathbf{y})$ defined for $\mathbf{y} \in \mathcal{Y}_{F}$, but for simplicity, we only consider the total external potential energy for $2 N$-periodic dead loads $\mathbf{f}$

$$
\mathcal{F}(\mathbf{y}):=-\sum_{\ell=-N+1}^{N} \epsilon f_{\ell} y_{\ell} .
$$

The equilibrium solution $\mathbf{y}^{a}$ of the EAM-atomistic model (3.1) then satisfies

$$
-\left\langle\delta \mathcal{E}^{a}\left(\mathbf{y}^{a}\right), \mathbf{w}\right\rangle=-\left\langle\delta \hat{\mathcal{E}}^{a}\left(\mathbf{y}^{a}\right), \mathbf{w}\right\rangle-\left\langle\delta \tilde{\mathcal{E}}^{a}\left(\mathbf{y}^{a}\right), \mathbf{w}\right\rangle=\left\langle\delta \mathcal{F}\left(\mathbf{y}^{a}\right), \mathbf{w}\right\rangle \quad \text { for all } \mathbf{w} \in \mathcal{U} .
$$

Here the negative of the embedding force of (3.3) is

$$
\begin{array}{r}
\left\langle\delta \hat{\mathcal{E}}^{a}\left(\mathbf{y}^{a}\right), \mathbf{w}\right\rangle=\epsilon \sum_{\ell=-N+1}^{N} G^{\prime}\left(\bar{\rho}_{\ell}^{a}\left(\mathbf{y}^{a}\right)\right) \cdot\left[\rho^{\prime}\left(D y_{\ell}^{a}\right) w_{\ell}^{\prime}+\rho^{\prime}\left(D y_{\ell}^{a}+D y_{\ell-1}^{a}\right)\left(w_{\ell}^{\prime}+w_{\ell-1}^{\prime}\right)\right. \\
\left.+\rho^{\prime}\left(D y_{\ell+1}^{a}\right) w_{\ell+1}^{\prime}+\rho^{\prime}\left(D y_{\ell+1}^{a}+D y_{\ell+2}^{a}\right)\left(w_{\ell+1}^{\prime}+w_{\ell+2}^{\prime}\right)\right],
\end{array}
$$

the negative of the pair potential force of (3.3) is given by

$$
\begin{aligned}
\left\langle\delta \tilde{\mathcal{E}}^{a}\left(\mathbf{y}^{a}\right), \mathbf{w}\right\rangle=\epsilon \sum_{\ell=-N+1}^{N} \frac{1}{2}\left[\phi^{\prime}\left(D y_{\ell}^{a}\right) w_{\ell}^{\prime}\right. & +\phi^{\prime}\left(D y_{\ell}^{a}+D y_{\ell-1}^{a}\right)\left(w_{\ell}^{\prime}+w_{\ell-1}^{\prime}\right) \\
& \left.+\phi^{\prime}\left(D y_{\ell+1}^{a}\right) w_{\ell+1}^{\prime}+\phi^{\prime}\left(D y_{\ell+1}^{a}+D y_{\ell+2}^{a}\right)\left(w_{\ell+1}^{\prime}+w_{\ell+2}^{\prime}\right)\right]
\end{aligned}
$$

and the negative of the external force is formulated as

$$
\langle\delta \mathcal{F}(\mathbf{y}), \mathbf{w}\rangle=\sum_{\ell=-N+1}^{N} \frac{\partial \mathcal{F}}{\partial y_{\ell}}(\mathbf{y}) w_{\ell}=-\sum_{\ell=-N+1}^{N} \epsilon f_{\ell} w_{\ell} .
$$

3.2. The Local EAM Approximations. In this subsection, we will briefly review the idea of the two different local approximations, the volume-based and the reconstruction-based, and give their expressions respectively.

3.2.1. The Volume-Based Local EAM Approximation. The idea of the volume-based local approximation based on the Cauchy-Born rule was first proposed in [12, 15, 17. We denote this energy by $\mathcal{E}^{c, v}(\mathbf{y})$, and we can formulate the local energy associated with each atom as

$$
\begin{aligned}
\mathcal{E}_{\ell}^{c, v}(\mathbf{y}):=\hat{\mathcal{E}}_{\ell}^{c, v}(\mathbf{y})+\tilde{\mathcal{E}}_{\ell}^{c, v}(\mathbf{y})=\frac{1}{2} & \left.G\left(\bar{\rho}_{\ell}^{c, v}(\mathbf{y})\right)\right)+\frac{1}{2} G\left(\bar{\rho}_{\ell+1}^{c, v}(\mathbf{y})\right) \\
& +\frac{1}{2}\left[\phi\left(y_{\ell}^{\prime}\right)+\phi\left(2 y_{\ell}^{\prime}\right)+\phi\left(y_{\ell+1}^{\prime}\right)+\phi\left(2 y_{\ell+1}^{\prime}\right)\right],
\end{aligned}
$$

where the total local electron density at atom $\ell$ is

$$
\bar{\rho}_{\ell}^{c, v}(\mathbf{y}):=2 \rho\left(y_{\ell}^{\prime}\right)+2 \rho\left(2 y_{\ell}^{\prime}\right) .
$$

Then the total volume-based local energy is

$$
\mathcal{E}_{\text {tot }}^{c, v}(\mathbf{y}):=\mathcal{E}^{c, v}(\mathbf{y})+\mathcal{F}(\mathbf{y})=\epsilon \sum_{\ell=-N+1}^{N} \mathcal{E}_{\ell}^{c, v}(\mathbf{y})-\epsilon \sum_{\ell=-N+1}^{N} f_{\ell} y_{\ell} .
$$

The equilibrium solution $\mathbf{y}^{c, v}$ then satisfies

$$
-\left\langle\delta \mathcal{E}^{c, v}\left(\mathbf{y}^{c, v}\right), \mathbf{w}\right\rangle=-\left\langle\delta \hat{\mathcal{E}}^{c, v}\left(\mathbf{y}^{c, v}\right), \mathbf{w}\right\rangle-\left\langle\delta \tilde{\mathcal{E}}^{c, v}\left(\mathbf{y}^{c, v}\right), \mathbf{w}\right\rangle=\left\langle\delta \mathcal{F}\left(\mathbf{y}^{c, v}\right), \mathbf{w}\right\rangle \quad \text { for all } \mathbf{w} \in \mathcal{U} .
$$


The negative of the embedding force of (3.5) is

$$
\begin{aligned}
\left\langle\delta \hat{\mathcal{E}}^{c, v}\left(\mathbf{y}^{c, v}\right), \mathbf{w}\right\rangle=\epsilon & \sum_{\ell=-N+1}^{N}\left\{G^{\prime}\left(\bar{\rho}_{\ell}^{c, v}\left(\mathbf{y}^{c, v}\right)\right) \cdot\left[\rho^{\prime}\left(D y_{\ell}^{c, v}\right)+2 \rho^{\prime}\left(2 D y_{\ell}^{c, v}\right)\right] w_{\ell}^{\prime}\right. \\
& \left.+G^{\prime}\left(\bar{\rho}_{\ell+1}^{c, v}\left(\mathbf{y}^{c, v}\right)\right) \cdot\left[\rho^{\prime}\left(D y_{\ell+1}^{c, v}\right)+2 \rho^{\prime}\left(2 D y_{\ell+1}^{c, v}\right)\right] w_{\ell+1}^{\prime}\right\},
\end{aligned}
$$

and the negative of the pair potential force of (3.5) is given by

$$
\left\langle\delta \tilde{\mathcal{E}}^{c, v}\left(\mathbf{y}^{c, v}\right), \mathbf{w}\right\rangle=\epsilon \sum_{\ell=-N+1}^{N} \frac{1}{2}\left\{\left[\phi^{\prime}\left(D y_{\ell}^{c, v}\right)+2 \phi^{\prime}\left(2 D y_{\ell}^{c, v}\right)\right] w_{\ell}^{\prime}+\left[\phi^{\prime}\left(D y_{\ell+1}^{c, v}\right)+2 \phi^{\prime}\left(2 D y_{\ell+1}^{c, v}\right)\right] w_{\ell+1}^{\prime}\right\} .
$$

3.2.2. The Reconstruction-Based Local EAM Approximation. Using the Cauchy-Born approximation, one can also reconstruct the position of each atom [5] and compute the energy $\mathcal{E}^{c, r}(\mathbf{y})$ by the approximation

$$
\mathcal{E}_{\ell}^{c, r}(\mathbf{y})=\hat{\mathcal{E}}_{\ell}^{c, r}(\mathbf{y})+\tilde{\mathcal{E}}_{\ell}^{c, r}(\mathbf{y})=G\left(\bar{\rho}_{\ell}^{c, r}(\mathbf{y})\right)+\frac{1}{2}\left[\phi\left(y_{\ell}^{\prime}\right)+\phi\left(2 y_{\ell}^{\prime}\right)+\phi\left(y_{\ell+1}^{\prime}\right)+\phi\left(2 y_{\ell+1}^{\prime}\right)\right],
$$

where the reconstruction-based local electron density at atom $\ell$ is

$$
\bar{\rho}_{\ell}^{c, r}(\mathbf{y}):=\rho\left(y_{\ell}^{\prime}\right)+\rho\left(2 y_{\ell}^{\prime}\right)+\rho\left(y_{\ell+1}^{\prime}\right)+\rho\left(2 y_{\ell+1}^{\prime}\right) .
$$

Thus, the total energy of the reconstruction-based local model is

$$
\begin{aligned}
\mathcal{E}_{\text {tot }}^{c, r}(\mathbf{y}):=\hat{\mathcal{E}}^{c, r}(\mathbf{y})+\tilde{\mathcal{E}}^{c, r}(\mathbf{y})+\mathcal{F}(\mathbf{y}) & \\
=\epsilon \sum_{\ell-N+1}^{N}\{ & G\left[\rho\left(y_{\ell}^{\prime}\right)+\rho\left(2 y_{\ell}^{\prime}\right)+\rho\left(y_{\ell+1}^{\prime}\right)+\rho\left(2 y_{\ell+1}^{\prime}\right)\right] \\
& \left.+\frac{1}{2}\left[\phi\left(y_{\ell}^{\prime}\right)+\phi\left(2 y_{\ell}^{\prime}\right)+\phi\left(y_{\ell+1}^{\prime}\right)+\phi\left(2 y_{\ell+1}^{\prime}\right)\right]\right\}-\epsilon \sum_{\ell=-N+1}^{N} f_{\ell} y_{\ell} .
\end{aligned}
$$

The volume-based and reconstruction-based local energies have the same pair potential energy, but their approximations for the embedding energy are quite different.

We compute the equilibrium solution of the reconstruction-based local model (3.6) from

$$
-\left\langle\delta \mathcal{E}^{c, r}\left(\mathbf{y}^{c, r}\right), \mathbf{w}\right\rangle=-\left\langle\delta \hat{\mathcal{E}}^{c, r}\left(\mathbf{y}^{c, r}\right), \mathbf{w}\right\rangle-\left\langle\delta \tilde{\mathcal{E}}^{c, r}\left(\mathbf{y}^{c, r}\right), \mathbf{w}\right\rangle=\left\langle\delta \mathcal{F}\left(\mathbf{y}^{c, r}\right), \mathbf{w}\right\rangle \quad \text { for all } \mathbf{w} \in \mathcal{U} .
$$

Here the negative of the embedding force of (3.7) is

$$
\begin{array}{r}
\left\langle\delta \hat{\mathcal{E}}^{c, r}\left(\mathbf{y}^{c, r}\right), \mathbf{w}\right\rangle=\epsilon \sum_{\ell=-N+1}^{N} G^{\prime}\left(\bar{\rho}_{\ell}^{c, r}\left(\mathbf{y}^{c, r}\right)\right) \cdot\left[\left(\rho^{\prime}\left(D y_{\ell}^{c, r}\right)+2 \rho^{\prime}\left(2 D y_{\ell}^{c, r}\right)\right) w_{\ell}^{\prime}\right. \\
\left.+\left(\rho^{\prime}\left(D y_{\ell+1}^{c, r}\right)+2 \rho^{\prime}\left(2 D y_{\ell+1}^{c, r}\right)\right) w_{\ell+1}^{\prime}\right],
\end{array}
$$

and the negative of the pair potential force of (3.7) is

$$
\left\langle\delta \tilde{\mathcal{E}}^{c, r}\left(\mathbf{y}^{c, r}\right), \mathbf{w}\right\rangle=\epsilon \sum_{\ell=-N+1}^{N} \frac{1}{2}\left\{\left[\phi^{\prime}\left(D y_{\ell}^{c, r}\right)+2 \phi^{\prime}\left(2 D y_{\ell}^{c, r}\right)\right] w_{\ell}^{\prime}+\left[\phi^{\prime}\left(D y_{\ell+1}^{c, r}\right)+2 \phi^{\prime}\left(2 D y_{\ell+1}^{c, r}\right)\right] w_{\ell+1}^{\prime}\right\} .
$$

The pair potential energy of both local approximations are exactly the same, but the embedding parts are quite different, which leads to different critical strains for lattice instability. We will analyze the lattice stability for all of the models in the next section. 


\section{Sharp Stability Analysis of The Atomistic and Local EAM Models.}

In this section, we analyze and compare the conditions for lattice stability of the atomistic model and the two local approximations for the next-nearest neighbor case. We will use techniques similar to those presented in [3] for the atomistic and quasicontinuum methods with pair potential interaction.

4.1. Stability of the Atomistic EAM Model. We first consider the fully atomistic model. The uniform deformation $\mathbf{y}_{F}$ is an equilibrium of the atomistic model (3.2) without external force. We call $\mathbf{y}_{F}$ stable in the atomistic model if and only if $\left\langle\delta^{2} \mathcal{E}^{a}\left(\mathbf{y}_{F}\right)\right.$ is positive definite, that is,

$$
\left\langle\delta^{2} \mathcal{E}^{a}\left(\mathbf{y}_{F}\right) \mathbf{u}, \mathbf{u}\right\rangle=\left\langle\delta^{2} \hat{\mathcal{E}}^{a}\left(\mathbf{y}_{F}\right) \mathbf{u}, \mathbf{u}\right\rangle+\left\langle\delta^{2} \tilde{\mathcal{E}}^{a}\left(\mathbf{y}_{F}\right) \mathbf{u}, \mathbf{u}\right\rangle>0 \quad \text { for all } \mathbf{u} \in \mathcal{U} \backslash\{\mathbf{0}\} .
$$

We computed $\left\langle\delta^{2} \tilde{\mathcal{E}}^{a}\left(\mathbf{y}_{F}\right) \mathbf{u}, \mathbf{u}\right\rangle$ in [3] to obtain

$$
\left\langle\delta^{2} \tilde{\mathcal{E}}^{a}\left(\mathbf{y}_{F}\right) \mathbf{u}, \mathbf{u}\right\rangle=\tilde{A}_{F}\|D \mathbf{u}\|_{\ell_{\epsilon}^{2}}^{2}-\epsilon^{2} \phi_{2 F}^{\prime \prime}\left\|D^{(2)} \mathbf{u}\right\|_{\ell_{\epsilon}^{2}}^{2},
$$

where

$$
\tilde{A}_{F}:=\phi_{F}^{\prime \prime}+4 \phi_{2 F}^{\prime \prime} \quad \text { for } \quad \phi_{F}^{\prime \prime}:=\phi^{\prime \prime}(F) \text { and } \phi_{2 F}^{\prime \prime}:=\phi^{\prime \prime}(2 F)
$$

is the continuum elastic modulus for the pair interaction potential. Thus, we focus on $\left\langle\delta^{2} \hat{\mathcal{E}}^{a}\left(\mathbf{y}_{F}\right) \mathbf{u}, \mathbf{u}\right\rangle$, which can be formulated as

$$
\left\langle\delta^{2} \hat{\mathcal{E}}^{a}\left(\mathbf{y}_{F}\right) \mathbf{u}, \mathbf{u}\right\rangle=\epsilon \sum_{\ell=-N+1}^{N}\left\{\begin{array}{c}
G_{F}^{\prime \prime}\left[\rho_{F}^{\prime}\left(u_{\ell}^{\prime}+u_{\ell+1}^{\prime}\right)+\rho_{2 F}^{\prime}\left(u_{\ell-1}^{\prime}+u_{\ell}^{\prime}+u_{\ell+1}^{\prime}+u_{\ell+2}^{\prime}\right)\right]^{2} \\
+G_{F}^{\prime}\left[\rho_{F}^{\prime \prime}\left(u_{\ell}^{\prime}\right)^{2}+\rho_{2 F}^{\prime \prime}\left(u_{\ell}^{\prime}+u_{\ell-1}^{\prime}\right)^{2}+\rho_{F}^{\prime \prime}\left(u_{\ell+1}^{\prime}\right)^{2}\right. \\
\left.\left.+\rho_{2 F}^{\prime \prime}\left(u_{\ell+1}^{\prime}+u_{\ell+2}^{\prime}\right)^{2}\right]\right\},
\end{array}\right.
$$

where we use the simplified notation

$$
\begin{gathered}
\rho_{F}^{\prime}:=\rho^{\prime}(F), \quad \rho_{F}^{\prime \prime}:=\rho^{\prime \prime}(F), \quad \rho_{2 F}^{\prime}:=\rho(2 F), \quad \rho_{2 F}^{\prime \prime}:=\rho^{\prime \prime}(2 F), \\
G_{F}^{\prime}:=G^{\prime}\left(\bar{\rho}_{\ell}^{a}\left(\mathbf{y}_{F}\right)\right)=G^{\prime}\left(\bar{\rho}_{\ell}^{c, v}\left(\mathbf{y}_{F}\right)\right)=G^{\prime}\left(\bar{\rho}_{\ell}^{c, r}\left(\mathbf{y}_{F}\right)\right), \\
G_{F}^{\prime \prime}:=G^{\prime \prime}\left(\bar{\rho}_{\ell}^{a}\left(\mathbf{y}_{F}\right)\right)=G^{\prime \prime}\left(\bar{\rho}_{\ell}^{c, v}\left(\mathbf{y}_{F}\right)\right)=G^{\prime \prime}\left(\bar{\rho}_{\ell}^{c, r}\left(\mathbf{y}_{F}\right)\right) .
\end{gathered}
$$

We define the continuum elastic modulus for the embedding energy to be

$$
\hat{A}_{F}:=4 G_{F}^{\prime \prime}\left(\rho_{F}^{\prime}+2 \rho_{2 F}^{\prime}\right)^{2}+2 G_{F}^{\prime}\left(\rho_{F}^{\prime \prime}+4 \rho_{2 F}^{\prime \prime}\right),
$$

and we define

$$
\begin{array}{r}
A_{F}:=\hat{A}_{F}+\tilde{A}_{F}, \quad B_{F}:=-\left[\phi_{2 F}^{\prime \prime}+G_{F}^{\prime \prime}\left(\left(\rho_{F}^{\prime}\right)^{2}+20\left(\rho_{2 F}^{\prime}\right)^{2}+12 \rho_{F}^{\prime} \rho_{2 F}^{\prime}\right)+G_{F}^{\prime}\left(2 \rho_{2 F}^{\prime \prime}\right)\right], \\
C_{F}:=G_{F}^{\prime \prime}\left(8\left(\rho_{2 F}^{\prime}\right)^{2}+2 \rho_{F}^{\prime} \rho_{2 F}^{\prime}\right), \text { and } D_{F}:=-G_{F}^{\prime \prime}\left(\rho_{2 F}^{\prime}\right)^{2} .
\end{array}
$$

Then (4.1) becomes

$$
\left\langle\delta^{2} \mathcal{E}^{a}\left(\mathbf{y}_{F}\right) \mathbf{u}, \mathbf{u}\right\rangle=A_{F}\|D \mathbf{u}\|_{\ell_{\epsilon}^{2}}^{2}+\epsilon^{2} B_{F}\left\|D^{(2)} \mathbf{u}\right\|_{\ell_{\epsilon}^{2}}^{2}+\epsilon^{4} C_{F}\left\|D^{(3)} \mathbf{u}\right\|_{\ell_{\epsilon}^{2}}^{2}+\epsilon^{6} D_{F}\left\|D^{(4)} \mathbf{u}\right\|_{\ell_{\epsilon}^{2}}^{2},
$$

where the detailed calculation can be found in the paper 10 .

We will analyze the stability of $\left\langle\delta^{2} \mathcal{E}^{a}\left(\mathbf{y}_{F}\right) \mathbf{u}, \mathbf{u}\right\rangle$ by using the Fourier representation 8]

$$
D u_{\ell}=\sum_{\substack{k=-N+1 \\ k \neq 0}}^{N} \frac{c_{k}}{\sqrt{2}} \cdot \exp \left(i k \frac{\ell}{N} \pi\right) .
$$


We exclude $k=0$ since $D \mathbf{u}$ must satisfy the mean zero condition $\sum_{\ell=-N+1}^{N} D u_{\ell}=0$.

It then follows from the discrete orthogonality of the Fourier basis that

$$
\begin{aligned}
\left\langle\delta^{2} \mathcal{E}^{a}\left(\mathbf{y}_{F}\right) \mathbf{u}, \mathbf{u}\right\rangle=\sum_{\substack{k=-N+1 \\
k \neq 0}}^{N}\left|c_{k}\right|^{2} \cdot\left\{A_{F}+B_{F}\left[4 \sin ^{2}\left(\frac{k \pi}{2 N}\right)\right]\right. & \\
+ & \left.C_{F}\left[4 \sin ^{2}\left(\frac{k \pi}{2 N}\right)\right]^{2}+D_{F}\left[4 \sin ^{2}\left(\frac{k \pi}{2 N}\right)\right]^{3}\right\} .
\end{aligned}
$$

We see from (4.9) that the eigenvalues $\lambda_{k}^{a}$ for $k=1, \ldots, N$ of $\left\langle\delta^{2} \mathcal{E}^{a}\left(\mathbf{y}_{F}\right) \mathbf{u}, \mathbf{u}\right\rangle$ with respect to the $\|D \mathbf{u}\|_{\ell_{\epsilon}^{2}}$ norm are given by

$$
\lambda_{k}^{a}=\lambda_{F}^{a}\left(s_{k}\right) \quad \text { for } \quad s_{k}=4 \sin ^{2}\left(\frac{k \pi}{2 N}\right)
$$

where

$$
\lambda_{F}^{a}(s):=A_{F}+B_{F} s+C_{F} s^{2}+D_{F} s^{3} .
$$

The energy and electron densities figures in [7] and [14 satisfy the following conditions which we shall assume in our analysis

$$
\phi_{F}^{\prime \prime}>0, \phi_{2 F}^{\prime \prime}<0 ; \quad \rho_{F}^{\prime} \leq 0, \rho_{2 F}^{\prime} \leq 0 ; \quad \rho_{F}^{\prime \prime} \geq 0, \rho_{2 F}^{\prime \prime} \geq 0 ; \quad \text { and } \quad G_{F}^{\prime \prime} \geq 0 .
$$

We can derive from the assumption (4.10) that

$$
C_{F}>0, \quad D_{F}<0, \quad \text { and } \quad 8\left|D_{F}\right| \leq C_{F} .
$$

Since (4.11) implies that $\left|D_{F} s\right| \leq 4\left|D_{F}\right| \leq C_{F} / 2$, for $0 \leq s \leq 4$, we have that

$$
\lambda_{F}^{a \prime}(s)=B_{F}+2 C_{F} s+3 D_{F} s^{2} \geq B_{F}+\frac{C_{F}}{2} s \text { for all } 0 \leq s \leq 4 .
$$

We note from (4.12) that the condition $B_{F} \geq 0$ or equivalently

$$
\phi_{2 F}^{\prime \prime}+G_{F}^{\prime \prime}\left[\left(\rho_{F}^{\prime}\right)^{2}+20\left(\rho_{2 F}^{\prime}\right)^{2}+12 \rho_{F}^{\prime} \rho_{2 F}^{\prime}\right]+G_{F}^{\prime} 2 \rho_{2 F}^{\prime \prime}=-B_{F} \leq 0,
$$

implies that $\lambda_{F}^{a}(s)$ is increasing for $0 \leq s \leq 4$. We thus conclude that if $B_{F} \geq 0$, then

$$
\left\langle\delta^{2} \mathcal{E}^{a}\left(\mathbf{y}_{F}\right) \mathbf{u}, \mathbf{u}\right\rangle \geq \lambda_{F}^{a}\left(s_{1}\right)\|D \mathbf{u}\|_{\ell_{\epsilon}^{2}}^{2} \geq\left(\hat{A}_{F}+\tilde{A}_{F}\right)\|D \mathbf{u}\|_{\ell_{\epsilon}^{2}}^{2} \quad \text { for all } \mathbf{u} \in \mathcal{U} .
$$

This result is summarized in the following theorem:

Theorem 4.1. Suppose that the hypotheses (4.10) and $B_{F} \geq 0$ hold. Then the uniform deformation $\mathbf{y}_{F}$ is stable for the atomistic model if and only if

$$
\begin{aligned}
\lambda_{F}^{a}\left(s_{1}\right)= & A_{F}+B_{F}\left[4 \sin ^{2}\left(\frac{\pi}{2 N}\right)\right]+C_{F}\left[4 \sin ^{2}\left(\frac{\pi}{2 N}\right)\right]^{2}+D_{F}\left[4 \sin ^{2}\left(\frac{\pi}{2 N}\right)\right]^{3} \\
= & \hat{A}_{F}+\tilde{A}_{F}-4 \sin ^{2}\left(\frac{\pi}{2 N}\right)\left\{\phi_{2 F}^{\prime \prime}+G_{F}^{\prime \prime}\left[\left(\rho_{F}^{\prime}\right)^{2}+20\left(\rho_{2 F}^{\prime}\right)^{2}+12 \rho_{F}^{\prime} \rho_{2 F}^{\prime}\right]+G_{F}^{\prime} 2 \rho_{2 F}^{\prime \prime}\right\} \\
& +4^{2} \sin ^{4}\left(\frac{\pi}{2 N}\right) G_{F}^{\prime \prime}\left[\eta\left(\rho_{2 F}^{\prime}\right)^{2}+2 \rho_{F}^{\prime} \rho_{2 F}^{\prime}\right]-4^{3} \sin ^{6}\left(\frac{\pi}{2 N}\right) G_{F}^{\prime \prime}\left(\rho_{2 F}^{\prime}\right)^{2}>0 .
\end{aligned}
$$

We note that the differences between $s_{k}$ and $s_{k-1}$ and between $\lambda_{F}^{a}\left(s_{k}\right)$ and $\lambda_{F}^{a}\left(s_{k-1}\right)$ are of order $O\left(\frac{2 k \pi^{2}}{4 N^{2}}\right)=O\left(2 k \epsilon^{2}\right)$ for $k=1, \ldots, N$. When the number of atoms $N$ is sufficiently large, $\min _{0 \leq s \leq 4} \lambda_{F}^{a}(s)$ can be used to approximate $\min _{1 \leq k \leq N} \lambda_{F}^{a}\left(s_{k}\right)$ with $1 \leq k \leq N$ with error at most of order $O(\epsilon)$ since $N \epsilon=1$. 
When $B_{F}<0$ and $N$ is sufficiently large, the minimum eigenvalue of $\delta^{2} \mathcal{E}^{a}\left(\mathbf{y}_{F}\right)$ is no longer $\lambda_{F}^{a}\left(s_{1}\right)$ and is given by the following theorem.

Theorem 4.2. Suppose that the hypotheses (4.10) and $B_{F}<0$ hold, and the number of atoms $N$ is sufficiently large. Then $\lambda_{F}^{a}\left(s_{1}\right)$ defined in Theorem 4.1] will no longer be the minimum eigenvalue of the second variation $\left\langle\delta^{2} \mathcal{E}^{a}\left(\mathbf{y}_{F}\right) \mathbf{u}, \mathbf{u}\right\rangle$. Instead, the minimum eigenvalue will be given by $\lambda_{F}^{a}\left(s_{k^{*}}\right)$ for some $s_{k^{*}}, 1<k^{*} \leq N$, that is either equal to 4 or close to

$$
s^{*}:=\frac{C_{F}-\sqrt{C_{F}^{2}-3 B_{F} D_{F}}}{-3 D_{F}}
$$

with difference of order $O\left(2 k^{*} \epsilon^{2}\right)$.

Proof. Here we will briefly discuss the role of the coefficient $B_{F}$ and leave the rigorous discussion of $\min _{0 \leq s \leq 4} \lambda_{F}^{a}(s)$ under the condition $B_{F}<0$ to section 5 ,

The assumption $B_{F} \geq 0$ guarantees that $u_{\ell}^{\prime}=\sin (\epsilon \ell \pi)$ is the eigenfunction corresponding to the minimum eigenvalue of $\delta^{2} \mathcal{E}^{a}\left(\mathbf{y}_{F}\right)$ with respect to the norm $\|D \mathbf{u}\|_{\ell_{\epsilon}^{2}}$. In fact, when $B_{F}<0$, we have $\lambda_{F}^{a}{ }^{\prime}(0)<0$ and thus $\lambda_{F}^{a}(0)$ will be strictly larger than $\lambda_{F}^{a}\left(s^{*}\right)$.

We note that the condition $B_{F} \geq 0$ cannot be expected to generally hold for EAM models when the nearest neighbor term $G_{F}^{\prime \prime}\left(\rho_{F}^{\prime}\right)^{2}>0$ dominates. We note, however, that generally $G_{F}^{\prime}<0$ for $F<1$ [14], in which case $B_{F} \geq 0$ is more likely to hold for compressive strains $F<1$.

Remark 4.1. We would like to point out that when $N$ is small, $\lambda_{F}^{a}\left(s_{1}\right)$ may be still the minimum eigenvalue of $\delta^{2} \mathcal{E}^{a}\left(\mathbf{y}_{F}\right)$ even if $B_{F}<0$. This is because $\lambda_{F}^{a}\left(s_{k}\right)$ is defined on the discrete domain $1 \leq k \leq N$, so the continuous function $\lambda_{F}^{a}(s)$ is not a good approximation unless $N$ is sufficiently large.

4.2. Stability of the Volume-Based and the Reconstruction-Based Local EAM Models. In this subsection, we will give stability estimations for the volume-based and the reconstructionbased local models, respectively.

4.2.1. Stability of the Volume-Based Local EAM Model. We focus on the stability of the volumebased local model under a uniform deformation $\mathbf{y}_{F}$. Using the equilibrium equation (3.5), we obtain the second variation $\delta^{2} \mathcal{E}^{c, v}\left(\mathbf{y}_{F}\right)$ for any $\mathbf{u} \in \mathcal{U} \backslash\{\mathbf{0}\}$

$$
\left\langle\delta^{2} \mathcal{E}^{c, v}\left(\mathbf{y}_{F}\right) \mathbf{u}, \mathbf{u}\right\rangle=\left(\hat{A}_{F}+\tilde{A}_{F}\right)\|D \mathbf{u}\|_{\ell_{\epsilon}^{2}}^{2}=A_{F}\|D \mathbf{u}\|_{\ell_{\epsilon}^{2}}^{2},
$$

where $\hat{A}_{F}$ and $\tilde{A}_{F}$ are defined in (4.5) and (4.3), respectively. It follows that $\mathbf{y}_{F}$ is stable in the volume-based local model if and only if $A_{F}:=\hat{A}_{F}+\tilde{A}_{F}>0$. We summarize this result in the following theorem.

Theorem 4.3. Suppose that the hypotheses (4.10) holds. Then the uniform deformation $\mathbf{y}_{F}$ is stable in the volume-based local model (3.4) if and only if $A_{F}:=\hat{A}_{F}+\tilde{A}_{F}>0$.

Remark 4.2. Comparing the conclusions in Theorem4.1 and Theorem4.3, we observe that when the hypothesis (4.13) is satisfied, the difference between the minimum eigenvalues of the fully atomistic and the volume-based local models is of order $O\left(\epsilon^{2}\right)$. This result is the same as for the pair potential case [4]. However, when the assumptions fails, the volume-based local model will be strictly more stable than the fully atomistic model, which will be discussed in the next remark. 
Remark 4.3. The assumption (4.13) is necessary for the validity of Theorem 4.1. We now give an explicit example showing that the uniform deformation can be strictly more stable for the volumebased local model (3.4) than for the fully atomistic model when (4.13) fails. We consider the case

$$
\phi_{2 F}^{\prime \prime}+G_{F}^{\prime \prime}\left(\rho_{F}^{\prime}+2 \rho_{2 F}^{\prime}\right)^{2}+G_{F}^{\prime} 2 \rho_{2 F}^{\prime \prime}>0 .
$$

Then (4.13) does not hold since it follows from (4.10) that

$$
\begin{aligned}
\phi_{2 F}^{\prime \prime} & +G_{F}^{\prime \prime}\left[\left(\rho_{F}^{\prime}\right)^{2}+20\left(\rho_{2 F}^{\prime}\right)^{2}+12 \rho_{F}^{\prime} \rho_{2 F}^{\prime}\right]+G_{F}^{\prime} 2 \rho_{2 F}^{\prime \prime} \\
& =\left[\phi_{2 F}^{\prime \prime}+G_{F}^{\prime \prime}\left(\rho_{F}^{\prime}+2 \rho_{2 F}^{\prime}\right)^{2}+G_{F}^{\prime} 2 \rho_{2 F}^{\prime \prime}\right]+8 G_{F}^{\prime \prime}\left(2\left(\rho_{2 F}^{\prime}\right)^{2}+\rho_{F}^{\prime} \rho_{2 F}^{\prime}\right) \\
& >0 .
\end{aligned}
$$

We define an oscillatory displacement $\tilde{\mathbf{u}}$, corresponding to the $k=N$ eigenmode in the Fourier expansion (4.8), by

$$
\tilde{u}_{\ell}:=(-1)^{\ell} \epsilon /(2 \sqrt{2})
$$

Therefore,

$$
\tilde{u}_{\ell}^{\prime}=(-1)^{\ell} /(\sqrt{2}), \quad\|D \tilde{\mathbf{u}}\|_{\ell_{\epsilon}^{2}}=1, \quad \tilde{u}_{\ell}^{\prime \prime}=(-1)^{\ell}(\sqrt{2}) / \epsilon .
$$

From (4.2) and (4.4) we can get

$$
\begin{aligned}
\left\langle\delta^{2} \mathcal{E}^{a}\left(\mathbf{y}_{F}\right) \tilde{\mathbf{u}}, \tilde{\mathbf{u}}\right\rangle & =\left\langle\delta^{2} \tilde{\mathcal{E}}^{a}\left(\mathbf{y}_{F}\right) \tilde{\mathbf{u}}, \tilde{\mathbf{u}}\right\rangle+\left\langle\delta^{2} \tilde{\mathcal{E}}^{a}\left(\mathbf{y}_{F}\right) \tilde{\mathbf{u}}, \tilde{\mathbf{u}}\right\rangle \\
& =\epsilon \sum_{\ell=-N+1}^{N} G_{F}^{\prime} 2 \rho_{F}^{\prime \prime} \frac{1}{2}+\left(\phi_{F}^{\prime \prime}+4 \phi_{2 F}^{\prime \prime}\right)\|D \tilde{\mathbf{u}}\|_{\ell_{\epsilon}^{2}}^{2}+\left(-\epsilon^{2} \phi_{2 F}^{\prime \prime}\right)\left\|D^{(2)} \tilde{\mathbf{u}}\right\|_{\ell_{\epsilon}^{2}}^{2} \\
& =G_{F}^{\prime} 2 \rho_{F}^{\prime \prime}+\left(\phi_{F}^{\prime \prime}+4 \phi_{2 F}^{\prime \prime}\right)-4 \phi_{2 F}^{\prime \prime}=\phi_{F}^{\prime \prime}+G_{F}^{\prime} 2 \rho_{F}^{\prime \prime} .
\end{aligned}
$$

Thus, we can obtain

$$
\inf _{\mathbf{u} \in \mathcal{U} \backslash\{\mathbf{0}\},\|D \mathbf{u}\|_{\ell_{\epsilon}^{2}}=1}\left\langle\delta^{2} \mathcal{E}^{a}\left(\mathbf{y}_{F}\right) \mathbf{u}, \mathbf{u}\right\rangle \leq \phi_{F}^{\prime \prime}+G_{F}^{\prime} 2 \rho_{F}^{\prime \prime} .
$$

On the other hand, from Theorem 4.3 we have that

$\inf _{\mathbf{u} \in \mathcal{U} \backslash\{\mathbf{0}\},\|D \mathbf{u}\|_{\ell_{\epsilon}^{2}}=1}\left\langle\delta^{2} \mathcal{E}^{c, v}\left(\mathbf{y}_{F}\right) \mathbf{u}, \mathbf{u}\right\rangle \equiv \tilde{A}_{F}+\tilde{A}_{F}=4\left[\phi_{2 F}^{\prime \prime}+G_{F}^{\prime \prime}\left(\rho_{F}^{\prime}+2 \rho_{2 F}^{\prime}\right)^{2}+G_{F}^{\prime} 2 \rho_{2 F}^{\prime \prime}\right]+\phi_{F}^{\prime \prime}+G_{F}^{\prime} 2 \rho_{F}^{\prime \prime}$.

Therefore, from (4.15) and (4.16) we have

$$
\inf _{\mathbf{u} \in \mathcal{U} \backslash\{\mathbf{0}\},\|D \mathbf{u}\|_{\ell_{\epsilon}^{2}}=1}\left\langle\delta^{2} \mathcal{E}^{c, v}\left(\mathbf{y}_{F}\right) \mathbf{u}, \mathbf{u}\right\rangle>\phi_{F}^{\prime \prime}+G_{F}^{\prime} 2 \rho_{F}^{\prime \prime} \geq \inf _{\mathbf{u} \in \mathcal{U} \backslash\{\mathbf{0}\},\|D \mathbf{u}\|_{\ell_{\epsilon}^{2}}=1}\left\langle\delta^{2} \mathcal{E}^{a}\left(\mathbf{y}_{F}\right) \mathbf{u}, \mathbf{u}\right\rangle .
$$

This inequality indicates that when the assumption (4.13) fails, the uniform deformation $\mathbf{y}_{F}$ can be unstable for the atomistic model, but still stable for the volume-based local model.

4.2.2. Stability of the Reconstruction-Based Local EAM Model. In this case, we do a similar calculation for the reconstruction-based local model and derive the second variation $\delta^{2} \mathcal{E}^{c, r}(\mathbf{y})$ from the 
equilibrium equation given by (3.7)

$$
\begin{aligned}
&\left\langle\delta^{2} \mathcal{E}^{c, r}\left(\mathbf{y}_{F}\right) \mathbf{u}, \mathbf{u}\right\rangle=\epsilon \sum_{\ell=-N+1}^{N}\left\{G_{F}^{\prime \prime}\left(\rho_{F}^{\prime}+2 \rho_{2 F}^{\prime}\right)^{2}\left(u_{\ell}^{\prime}+u_{\ell+1}^{\prime}\right)^{2}+G_{F}^{\prime}\left(\rho_{F}^{\prime \prime}+4 \rho_{2 F}^{\prime \prime}\right)\left[\left(u_{\ell}^{\prime}\right)^{2}+\left(u_{\ell+1}^{\prime}\right)^{2}\right]\right\} \\
& \quad+\epsilon \sum_{\ell=-N+1}^{N} \frac{1}{2}\left\{\phi_{F}^{\prime \prime}\left[\left(u_{\ell}^{\prime}\right)^{2}+\left(u_{\ell+1}^{\prime}\right)^{2}\right]+\phi_{2 F}^{\prime \prime}\left[\left(4 u_{\ell}^{\prime}\right)^{2}+4\left(u_{\ell+1}^{\prime}\right)^{2}\right]\right\} \\
&=\left[4 G_{F}^{\prime \prime}\left(\rho_{F}^{\prime}+2 \rho_{2 F}^{\prime}\right)^{2}+2 G_{F}^{\prime}\left(\rho_{F}^{\prime \prime}+4 \rho_{2 F}^{\prime \prime}\right)+\phi_{F}^{\prime \prime}+4 \phi_{2 F}^{\prime \prime}\right]\|D \mathbf{u}\|_{\ell_{\epsilon}^{2}}^{2} \\
& \quad-\epsilon^{2} G_{F}^{\prime \prime}\left(\rho_{F}^{\prime}+2 \rho_{2 F}^{\prime}\right)^{2}\left\|D^{(2)} \mathbf{u}\right\|_{\ell_{\epsilon}^{2}}^{2} \\
&=A_{F}\|D \mathbf{u}\|_{\ell_{\epsilon}^{2}}^{2}+\epsilon^{2} \tilde{B}_{F}\left\|D^{(2)} \mathbf{u}\right\|_{\ell_{\epsilon}^{2}}^{2},
\end{aligned}
$$

where $A_{F}$ is defined in (4.3) and $\tilde{B}_{F}$ is defined to be

$$
\tilde{B}_{F}:=-G_{F}^{\prime \prime}\left(\rho_{F}^{\prime}+2 \rho_{2 F}^{\prime}\right)^{2} \leq 0 .
$$

We recall that for the EAM-atomistic model, the coefficient $B_{F}$ of $\|D \mathbf{u}\|_{\ell_{\epsilon}^{2}}^{2}$ in (4.7) is defined as

$$
B_{F}=-\left[\phi_{2 F}^{\prime \prime}+G_{F}^{\prime \prime}\left(\left(\rho_{F}^{\prime}\right)^{2}+20\left(\rho_{2 F}^{\prime}\right)^{2}+12 \rho_{F}^{\prime} \rho_{2 F}^{\prime}\right)+G_{F}^{\prime}\left(2 \rho_{2 F}^{\prime \prime}\right)\right] .
$$

Comparing $B_{F}$ with $\tilde{B}_{F}$ defined in (4.17), we find that

$$
B_{F}=\tilde{B}_{F}-\left[\phi_{2 F}^{\prime \prime}+G_{F}^{\prime \prime}\left(16\left(\rho_{2 F}^{\prime}\right)^{2}+8 \rho_{F}^{\prime} \rho_{2 F}^{\prime}\right)+G_{F}^{\prime}\left(2 \rho_{2 F}^{\prime \prime}\right)\right] .
$$

The assumption (4.10) that $\phi_{2 F}^{\prime \prime}<0$ implies $B_{F}$ can be positive while $\tilde{B}_{F}$ is always negative.

We similarly use the Fourier representation

$$
D u_{\ell}=\sum_{\substack{k=-N+1 \\ k \neq 0}}^{N} \frac{c_{k}}{\sqrt{2}} \cdot \exp \left(i k \frac{\ell}{N} \pi\right)
$$

to analyze the stability of $\delta^{2} \mathcal{E}^{c, r}\left(\mathbf{y}_{F}\right)$. Again, we exclude $k=0$ because of the mean zero condition of $D \mathbf{u}$. From the discrete orthogonality of the Fourier basis we have

$$
\left\langle\delta^{2} \mathcal{E}^{c, r}\left(\mathbf{y}_{F}\right) \mathbf{u}, \mathbf{u}\right\rangle=\sum_{\substack{k=-N+1 \\ k \neq 0}}^{N}\left|c_{k}\right|^{2} \cdot\left\{A_{F}+\tilde{B}_{F}\left[4 \sin ^{2}\left(\frac{k \pi}{2 N}\right)\right]\right\} .
$$

The eigenvalues $\lambda_{k}^{c, r}$ of $\left\langle\delta^{2} \mathcal{E}^{c, r}\left(\mathbf{y}_{F}\right) \mathbf{u}, \mathbf{u}\right\rangle$ with respect to the $\|D \mathbf{u}\|_{\ell_{\epsilon}^{2}}$ norm are given by

$$
\lambda_{k}^{c, r}=\lambda_{F}^{c, r}\left(s_{k}\right) \quad \text { for } k=1, \ldots, N
$$

where

$$
s_{k}=4 \sin ^{2}\left(\frac{k \pi}{2 N}\right) \quad \text { and } \quad \lambda_{F}^{c, r}(s):=A_{F}+\tilde{B}_{F} s .
$$

The assumption (4.10) implies that $\tilde{B}_{F} \leq 0$ always holds, so $\lambda_{F}^{c, r}(s)$ is decreasing for $0 \leq s \leq 4$ and the minimum eigenvalue of $\delta^{2} \mathcal{E}^{c, r}\left(\mathbf{y}_{F}\right)$ is achieved at $k=N$, i.e, $s_{N}=4$ :

$$
\min _{1 \leq k \leq N} \lambda_{F}^{c, r}\left(s_{k}\right)=\lambda_{F}^{c, r}(4)=A_{F}+4 \tilde{B}_{F}=2 G_{F}^{\prime}\left(\rho_{F}^{\prime \prime}+4 \rho_{2 F}^{\prime \prime}\right)+\phi_{F}^{\prime \prime}+4 \phi_{2 F}^{\prime \prime} .
$$


The minimum eigenmode is given by the oscillatory displacement $\hat{u}_{\ell}^{\prime}=-\hat{u}_{\ell+1}^{\prime}$ since

$$
\left\langle\delta^{2} \mathcal{E}^{c, r}\left(\mathbf{y}_{F}\right) \hat{\mathbf{u}}, \hat{\mathbf{u}}\right\rangle=\left[2 G_{F}^{\prime}\left(\rho_{F}^{\prime \prime}+4 \rho_{2 F}^{\prime \prime}\right)+\phi_{F}^{\prime \prime}+4 \phi_{2 F}^{\prime \prime}\right]\|D \hat{\mathbf{u}}\|_{\ell_{\epsilon}^{2}}^{2} .
$$

We thus have the following stability result for the reconstruction-based local model.

Theorem 4.4. Suppose that the hypotheses (4.10) holds. Then the uniform deformation $\mathbf{y}_{F}$ is stable in the reconstruction-based local model (3.6) if and only if

$$
2 G_{F}^{\prime}\left(\rho_{F}^{\prime \prime}+4 \rho_{2 F}^{\prime \prime}\right)+\phi_{F}^{\prime \prime}+4 \phi_{2 F}^{\prime \prime}>0
$$

Remark 4.4. Comparing the conclusions in Theorem 4.1, Theorem 4.3, and Theorem 4.4, we note that when the assumption (4.13) holds, i.e., $B_{F} \geq 0$, the fully atomistic model is strictly more stable than the reconstruction-based local model. The difference between their minimum eigenvalues is $O(1)$, not $O\left(\epsilon^{2}\right)$ as for the volume-based approximation. When the assumption (4.13) fails, i.e. $B_{F}<0$, the conclusion will be different, and we will rigorously analyze this case in section 5 ,

\section{Comparison of the Stability of the Atomistic and Local EAM Models}

In this section, we would like to give a full discussion of the sharp stability estimates for all of the EAM models. Recall that the eigenvalue function of $\delta^{2} \mathcal{E}^{a}\left(\mathbf{y}_{F}\right)$ is

$$
\lambda_{F}^{a}\left(s_{k}\right):=A_{F}+B_{F} s_{k}+C_{F} s_{k}^{2}+D_{F} s_{k}^{3} \quad \text { for } \quad s_{k}=4 \sin ^{2}\left(\frac{k \pi}{2 N}\right), \quad k=1, \ldots, N,
$$

where the coefficients $A_{F}, B_{F}, C_{F}$ and $D_{F}$ are given in the equation (4.6).

To simplify the following analyses, the number of atoms $N$ is assumed to be sufficiently large. Thus, we use the global minimum of the continuous function $\lambda_{F}^{a}(s):=A_{F}+B_{F} s+C_{F} s^{2}+D_{F} s^{3}$ for $0 \leq s \leq 4$ to approximate $\min _{1 \leq k \leq N} \lambda_{F}^{a}\left(s_{k}\right)$. We note that their difference is at most of order $O\left(2 k \epsilon^{2}\right) \leq O(\epsilon)$.

We recall that

$$
\min _{0 \leq s \leq 4} \lambda_{F}^{a}(s)=\lambda_{F}^{a}(0) \quad \text { if } B_{F} \geq 0
$$

To find $\min _{0 \leq s \leq 4} \lambda_{F}^{a}(s)$ when $B_{F}<0$, we first evaluate $\lambda_{F}^{a}(s)$ at $s=0,4$ :

$$
\begin{aligned}
& \lambda_{F}^{a}(0)=A_{F}=4 G_{F}^{\prime \prime}\left(\rho_{F}^{\prime}+2 \rho_{2 F}^{\prime}\right)^{2}+2 G_{F}^{\prime}\left(\rho_{F}^{\prime \prime}+4 \rho_{2 F}^{\prime \prime}\right)+\phi_{F}^{\prime}+4 \phi_{2 F}^{\prime \prime}, \\
& \lambda_{F}^{a}(4)=\phi_{F}^{\prime \prime}+2 G_{F}^{\prime} \rho_{F}^{\prime \prime} .
\end{aligned}
$$

We next compute the first and second derivatives of $\lambda_{F}^{a}(s)$, which are

$$
\begin{aligned}
\lambda_{F}^{a \prime}(s) & =B_{F}+2 C_{F} s+3 D_{F} s^{2}, \\
\lambda_{F}^{a}{ }^{\prime \prime}(s) & =2 C_{F}+6 D_{F} s .
\end{aligned}
$$

Since $\lambda_{F}^{a}{ }^{\prime}(s)$ is a quadratic function, we thus have two critical points of $\lambda_{F}^{a}(s)$ when the coefficients satisfy

$$
C_{F}^{2}-3 B_{F} D_{F} \geq 0 \quad \text { or equivalently } \quad \phi_{2 F}^{\prime \prime}+2 G_{F}^{\prime} \rho_{2 F}^{\prime \prime} \leq \frac{1}{3} G_{F}^{\prime \prime}\left(\rho_{F}^{\prime}-2 \rho_{2 F}^{\prime}\right)^{2} .
$$

We can summarize the case when $B_{F}<0$ and $C_{F}^{2}-3 B_{F} D_{F} \leq 0$ by

$$
\min _{0 \leq s \leq 4} \lambda_{F}^{a}(s)=\lambda_{F}^{a}(4) \quad \text { if } B_{F}<0 \text { and } C_{F}^{2}-3 B_{F} D_{F} \leq 0 \text {. }
$$

In the case $C_{F}^{2}-3 B_{F} D_{F}>0$, the critical points are

$$
s_{1}=\frac{C_{F}-\sqrt{C_{F}^{2}-3 B_{F} D_{F}}}{-3 D_{F}} \quad \text { and } \quad s_{2}=\frac{C_{F}+\sqrt{C_{F}^{2}-3 B_{F} D_{F}}}{-3 D_{F}} .
$$


Since $D_{F}<0, \lambda_{F}^{a}(s)$ will then have a local minimum at $s^{*}=s_{1}$ and a local maximum at $s_{2}$. The corresponding local minimum value is

$$
\begin{aligned}
\lambda_{F}^{a}\left(s^{*}\right) & =A_{F}+B_{F} s^{*}+C_{F}\left(s^{*}\right)^{2}+D_{F}\left(s^{*}\right)^{3} \\
& =A_{F}+s^{*}\left(\frac{B_{F}}{2}-\frac{D_{F}}{2}\left(s^{*}\right)^{2}\right),
\end{aligned}
$$

where we use $\lambda_{F}^{a}{ }^{\prime}\left(s^{*}\right)=0$ to get the last equality. We can thus summarize all of the cases by

$$
\begin{aligned}
& \min _{0 \leq s \leq 4} \lambda_{F}^{a}(s)=\lambda_{F}^{a}(0) \quad \text { if } B_{F} \geq 0, \\
& \min _{0 \leq s \leq 4} \lambda_{F}^{a}(s)=\lambda_{F}^{a}(4) \quad \text { if } B_{F}<0 \text { and } C_{F}^{2}-3 B_{F} D_{F} \leq 0, \\
& \min _{0 \leq s \leq 4} \lambda_{F}^{a}(s)=\min \left\{\lambda_{F}^{a}\left(s^{*}\right), \lambda_{F}^{a}(4)\right\} \quad \text { if } B_{F}<0 \text { and } C_{F}^{2}-3 B_{F} D_{F}>0 .
\end{aligned}
$$

We note that the minimum eigenvalues of the volume-based and the reconstruction-based local models are separately given by the following expressions

$$
\begin{aligned}
& \min _{0 \leq s \leq 4} \lambda_{F}^{c, v}(s)=\lambda_{F}^{a}(0)=A_{F}=4 G_{F}^{\prime \prime}\left(\rho_{F}^{\prime}+2 \rho_{2 F}^{\prime}\right)^{2}+2 G_{F}^{\prime}\left(\rho_{F}^{\prime \prime}+4 \rho_{2 F}^{\prime \prime}\right)+\phi_{F}^{\prime \prime}+4 \phi_{2 F}^{\prime \prime}, \\
& \min _{0 \leq s \leq 4} \lambda_{F}^{c, r}(s)=\lambda_{F}^{c, r}(4)=A_{F}-4 G_{F}^{\prime \prime}\left(\rho_{F}^{\prime}+2 \rho_{2 F}^{\prime}\right)^{2}=2 G_{F}^{\prime}\left(\rho_{F}^{\prime \prime}+4 \rho_{2 F}^{\prime \prime}\right)+\phi_{F}^{\prime \prime}+4 \phi_{2 F}^{\prime \prime} .
\end{aligned}
$$

5.1. The Volume-based Local EAM Model versus the Fully Atomistic EAM Model. We first compare the minimum eigenvalues of the volume-based local and the fully atomistic models. Combining the results of Theorem 4.1 and Theorem 4.2, we have the following theorem.

Theorem 5.1. The relation of the stability of the volume-based local model and the fully atomistic model depends on the sign of

$$
B_{F}:=-\left[\phi_{2 F}^{\prime \prime}+G_{F}^{\prime \prime}\left(\left(\rho_{F}^{\prime}\right)^{2}+20\left(\rho_{2 F}^{\prime}\right)^{2}+12 \rho_{F}^{\prime} \rho_{2 F}^{\prime}\right)+G_{F}^{\prime}\left(2 \rho_{2 F}^{\prime \prime}\right)\right]
$$

and can be summarized as follows:

$$
\begin{aligned}
& \min _{0 \leq s \leq 4} \lambda_{F}^{a}(s)=\lambda_{F}^{a}(0)=\lambda_{F}^{c, v} \quad \text { if } \quad B_{F} \geq 0, \\
& \min _{0 \leq s \leq 4} \lambda_{F}^{a}(s)=\min \left\{\lambda_{F}^{a}\left(s^{*}\right), \lambda_{F}^{a}(4)\right\}<\lambda_{F}^{a}(0)=\min _{0 \leq s \leq 4} \lambda_{F}^{c, v} \quad \text { if } \quad B_{F}<0 .
\end{aligned}
$$

This observation indicates that the set of stable uniform strains for the volume-based local model always includes that for the fully atomistic EAM model.

5.2. The Reconstruction-based Local EAM Model versus the Fully Atomistic EAM Model. The relation of the minimum eigenvalues for $\delta^{2} \mathcal{E}^{a}\left(\mathbf{y}_{F}\right)$ and $\delta^{2} \mathcal{E}^{c, r}\left(\mathbf{y}_{F}\right)$ is more complicated. We note that assumption (4.10) implies

$$
\begin{aligned}
\lambda_{F}^{a}(0) & =A_{F}=4 G_{F}^{\prime \prime}\left(\rho_{F}^{\prime}+2 \rho_{2 F}^{\prime}\right)^{2}+2 G_{F}^{\prime}\left(\rho_{F}^{\prime \prime}+4 \rho_{2 F}^{\prime \prime}\right)+\phi_{F}^{\prime \prime}+4 \phi_{2 F}^{\prime \prime} \\
& \geq 2 G_{F}^{\prime}\left(\rho_{F}^{\prime \prime}+4 \rho_{2 F}^{\prime \prime}\right)+\phi_{F}^{\prime \prime}+4 \phi_{2 F}^{\prime \prime}=\min _{0 \leq s \leq 4} \lambda_{F}^{c, r}(s),
\end{aligned}
$$

and we have

$$
\lambda_{F}^{a}(4)-\min _{0 \leq s \leq 4} \lambda_{F}^{c, r}(s)=-4\left(\phi_{2 F}^{\prime \prime}+G_{F}^{\prime} \cdot 2 \rho_{2 F}^{\prime \prime}\right) .
$$

We thus conclude that if

$$
\phi_{2 F}^{\prime \prime}+G_{F}^{\prime} \cdot 2 \rho_{2 F}^{\prime \prime} \leq 0,
$$


then

$$
\lambda_{F}^{a}(4) \geq \min _{0 \leq s \leq 4} \lambda_{F}^{c, r}(s) .
$$

The equal sign is achieved if and only if $\phi_{2 F}^{\prime \prime}+G_{F}^{\prime} \cdot 2 \rho_{2 F}^{\prime \prime}=0$. We also have the identity

$$
\phi_{2 F}^{\prime \prime}+G_{F}^{\prime} \cdot 2 \rho_{2 F}^{\prime \prime}=-B_{F}-\left[G_{F}^{\prime \prime}\left(\left(\rho_{F}^{\prime}\right)^{2}+20\left(\rho_{2 F}^{\prime}\right)^{2}+12 \rho_{F}^{\prime} \rho_{2 F}^{\prime}\right)\right] .
$$

We next compare $\lambda_{F}^{a}\left(s^{*}\right)$ and $\min _{0 \leq s \leq 4} \lambda_{F}^{c, r}(s)$. The difference of these two is

$$
\begin{aligned}
\lambda_{F}^{a}\left(s^{*}\right)- & \min _{0 \leq s \leq 4} \lambda_{F}^{c, r}(s) \\
= & 4 G_{F}^{\prime \prime}\left(\rho_{F}^{\prime}+2 \rho_{2 F}^{\prime}\right)^{2}+s^{*}\left(\frac{B_{F}}{2}-\frac{D_{F}}{2}\left(s^{*}\right)^{2}\right) \\
= & 4 G_{F}^{\prime \prime}\left(\rho_{F}^{\prime}+2 \rho_{2 F}^{\prime}\right)^{2} \\
& +\frac{C_{F}-\sqrt{C_{F}^{2}-3 B_{F} D_{F}}}{-3 D_{F}} \cdot \frac{6 B_{F} D_{F}-2 C_{F}^{2}+C_{F}\left(C_{F}+\sqrt{C_{F}^{2}-3 B_{F} D_{F}}\right)}{9 D_{F}} \\
= & 4 G_{F}^{\prime \prime}\left(\rho_{F}^{\prime}+2 \rho_{2 F}^{\prime}\right)^{2}+\frac{B_{F} C_{F}}{-9 D_{F}}+\frac{2\left(C_{F}^{2}-3 B_{F} D_{F}\right)\left(C_{F}-\sqrt{C_{F}^{2}-3 B_{F} D_{F}}\right)}{27 D_{F}^{2}} \\
\geq & 4 G_{F}^{\prime \prime}\left(\rho_{F}^{\prime}+2 \rho_{2 F}^{\prime}\right)^{2}+\frac{B_{F} C_{F}}{-9 D_{F}} .
\end{aligned}
$$

According to the assumption (5.5), we can use the definition of $C_{F}$ and $D_{F}$ (4.6) to get

$$
\begin{aligned}
B_{F} & =-\left[\phi_{2 F}^{\prime \prime}+G_{F}^{\prime} \cdot 2 \rho_{2 F}^{\prime \prime}+G_{F}^{\prime \prime}\left(\left(\rho_{F}^{\prime}\right)^{2}+12 \rho_{F}^{\prime} \rho_{2 F}^{\prime}+20\left(\rho_{2 F}^{\prime}\right)^{2}\right)\right] \\
& \geq-G_{F}^{\prime \prime}\left(\left(\rho_{F}^{\prime}\right)^{2}+12 \rho_{F}^{\prime} \rho_{2 F}^{\prime}+20\left(\rho_{2 F}^{\prime}\right)^{2}\right) .
\end{aligned}
$$

We thus can obtain from the above inequality and the assumption (4.10) that

$$
\begin{aligned}
\lambda_{F}^{a}\left(s^{*}\right)- & \min _{0 \leq s \leq 4} \lambda_{F}^{c, r}(s) \\
& \geq 4 G_{F}^{\prime \prime}\left(\rho_{F}^{\prime}+2 \rho_{2 F}^{\prime}\right)^{2}+\frac{B_{F} C_{F}}{-9 D_{F}} \\
& \geq 4 G_{F}^{\prime \prime}\left(\rho_{F}^{\prime}+2 \rho_{2 F}^{\prime}\right)^{2}+\frac{G_{F}^{\prime \prime}\left(8 \rho_{2 F}^{\prime}+2 \rho_{F}^{\prime}\right)\left(\left(\rho_{F}^{\prime}\right)^{2}+12 \rho_{F}^{\prime} \rho_{2 F}^{\prime}+20\left(\rho_{2 F}^{\prime}\right)^{2}\right)}{-9 \rho_{2 F}^{\prime}} \\
& =2 G_{F}^{\prime \prime} \frac{\left(\rho_{F}^{\prime}+2 \rho_{2 F}^{\prime}\right)\left(2 \rho_{2 F}^{\prime}-\rho_{F}^{\prime}\right)^{2}}{9 \rho_{2 F}^{\prime}} \geq 0 .
\end{aligned}
$$

Therefore, we have that

$$
\begin{gathered}
\min _{0 \leq s \leq 4} \lambda_{F}^{a}(s)=\min \left\{\lambda_{F}^{a}(0), \lambda_{F}^{a}\left(s^{*}\right), \lambda_{F}^{a}(4)\right\}=\min _{0 \leq s \leq 4} \lambda_{F}^{c, r}(s) \quad \text { if } \quad \phi_{2 F}^{\prime \prime}+G_{F}^{\prime} \cdot 2 \rho_{2 F}^{\prime \prime}=0, \\
\min _{0 \leq s \leq 4} \lambda_{F}^{a}(s)=\min \left\{\lambda_{F}^{a}(0), \lambda_{F}^{a}\left(s^{*}\right), \lambda_{F}^{a}(4)\right\}>\min _{0 \leq s \leq 4} \lambda_{F}^{c, r}(s) \quad \text { if } \quad \phi_{2 F}^{\prime \prime}+G_{F}^{\prime} \cdot 2 \rho_{2 F}^{\prime \prime}<0 .
\end{gathered}
$$

Now let us turn to the case that the assumption (5.5) fails, which means

$$
\phi_{2 F}^{\prime \prime}+G_{F}^{\prime} \cdot 2 \rho_{2 F}^{\prime \prime}>0 .
$$


In this case we have the opposite conclusion that the fully atomistic model $\mathcal{E}^{a}(\mathbf{y})$ is strictly less stable than the reconstruction-based local model $\mathcal{E}^{c, r}(\mathbf{y})$. From the condition (5.8), we have

$$
\begin{gathered}
\lambda_{F}^{a}(4)-\min _{0 \leq s \leq 4} \lambda_{F}^{c, r}(s)=-4\left(\phi_{2 F}^{\prime \prime}+G_{F}^{\prime} \cdot 2 \rho_{2 F}^{\prime \prime}\right)<0, \\
\text { i.e., } \quad \lambda_{F}^{a}(4)<\min _{0 \leq s \leq 4} \lambda_{F}^{c, r}(s) .
\end{gathered}
$$

Before comparing $\lambda_{F}^{a}\left(s^{*}\right)$ and $\min _{0 \leq s \leq 4} \lambda_{F}^{c, r}(s)$, we recall that $s^{*}$ exists if and only if

$$
\phi_{2 F}^{\prime \prime}+2 G_{F}^{\prime} \rho_{2 F}^{\prime \prime} \leq \frac{1}{3} G_{F}^{\prime \prime}\left(\rho_{F}^{\prime}-2 \rho_{2 F}^{\prime}\right)^{2} .
$$

Thus, we actually consider the case that

$$
0<\phi_{2 F}^{\prime \prime}+2 G_{F}^{\prime} \rho_{2 F}^{\prime \prime} \leq \frac{1}{3} G_{F}^{\prime \prime}\left(\rho_{F}^{\prime}-2 \rho_{2 F}^{\prime}\right)^{2} .
$$

We substitute $B_{F}=-\left[\phi_{2 F}^{\prime \prime}+G_{F}^{\prime} \cdot 2 \rho_{2 F}^{\prime \prime}+G_{F}^{\prime \prime}\left(\left(\rho_{F}^{\prime}\right)^{2}+12 \rho_{F}^{\prime} \rho_{2 F}^{\prime}+20\left(\rho_{2 F}^{\prime}\right)^{2}\right)\right]$ into the inequality (5.6) and get

$$
\begin{aligned}
\lambda_{F}^{a}\left(s^{*}\right)- & \min _{0 \leq s \leq 4} \lambda_{F}^{c, r}(s) \\
& \geq 4 G_{F}^{\prime \prime}\left(\rho_{F}^{\prime}+2 \rho_{2 F}^{\prime}\right)^{2}+\frac{B_{F} C_{F}}{-9 D_{F}} \\
& =4 G_{F}^{\prime \prime}\left(\rho_{F}^{\prime}+2 \rho_{2 F}^{\prime}\right)^{2}+\left(\frac{\phi_{2 F}^{\prime \prime}+G_{F}^{\prime} \cdot 2 \rho_{2 F}^{\prime \prime}}{-9 G_{F}^{\prime \prime}\left(\rho_{2 F}^{\prime}\right)^{2}}+\frac{G_{F}^{\prime \prime}\left(\left(\rho_{F}^{\prime}\right)^{2}+12 \rho_{F}^{\prime} \rho_{2 F}^{\prime}+20\left(\rho_{2 F}^{\prime}\right)^{2}\right)}{-9 G_{F}^{\prime \prime}\left(\rho_{2 F}^{\prime}\right)^{2}}\right) C_{F} \\
& =\frac{\left(\phi_{2 F}^{\prime \prime}+G_{F}^{\prime} \cdot 2 \rho_{2 F}^{\prime \prime}\right)\left(8 \rho_{2 F}^{\prime}+2 \rho_{F}^{\prime}\right)}{-9 \rho_{2 F}^{\prime}}+2 G_{F}^{\prime \prime} \frac{\left(\rho_{F}^{\prime}+2 \rho_{2 F}^{\prime}\right)\left(2 \rho_{2 F}^{\prime}-\rho_{F}^{\prime}\right)^{2}}{9 \rho_{2 F}^{\prime}} .
\end{aligned}
$$

Since $\phi_{2 F}^{\prime \prime}+2 G_{F}^{\prime} \rho_{2 F}^{\prime \prime} \leq \frac{1}{3} G_{F}^{\prime \prime}\left(\rho_{F}^{\prime}-2 \rho_{2 F}^{\prime}\right)^{2}$, therefore

$$
\begin{aligned}
\lambda_{F}^{a}\left(s^{*}\right)- & \min _{0 \leq s \leq 4} \lambda_{F}^{c, r}(s) \\
& \geq \frac{\left(\phi_{2 F}^{\prime \prime}+G_{F}^{\prime} \cdot 2 \rho_{2 F}^{\prime \prime}\right)\left(8 \rho_{2 F}^{\prime}+2 \rho_{F}^{\prime}\right)}{-9 \rho_{2 F}^{\prime}}+2 G_{F}^{\prime \prime} \frac{\left(\rho_{F}^{\prime}+2 \rho_{2 F}^{\prime}\right)\left(2 \rho_{2 F}^{\prime}-\rho_{F}^{\prime}\right)^{2}}{9 \rho_{2 F}^{\prime}} \\
& \geq \frac{\frac{1}{3} G_{F}^{\prime \prime}\left(\rho_{F}^{\prime}-2 \rho_{2 F}^{\prime}\right)^{2}\left(8 \rho_{2 F}^{\prime}+2 \rho_{F}^{\prime}\right)}{-9 \rho_{2 F}^{\prime}}+2 G_{F}^{\prime \prime} \frac{\left(\rho_{F}^{\prime}+2 \rho_{2 F}^{\prime}\right)\left(2 \rho_{2 F}^{\prime}-\rho_{F}^{\prime}\right)^{2}}{9 \rho_{2 F}^{\prime}} \\
& =\frac{1}{3} G_{F}^{\prime \prime}\left(\rho_{F}^{\prime}-2 \rho_{2 F}^{\prime}\right)^{2} \frac{4 \rho_{2 F}^{\prime}+4 \rho_{F}^{\prime}}{9 \rho_{2 F}^{\prime}} \geq 0 .
\end{aligned}
$$

Hence, when $s^{*}$ exists and the assumption (5.8) holds, we have

$$
\min _{0 \leq s \leq 4} \lambda_{F}^{a}(s)=\min \left\{\lambda_{F}^{a}(0), \lambda_{F}^{a}\left(s^{*}\right), \lambda_{F}^{a}(4)\right\}=\lambda_{F}^{a}(4)<\min _{0 \leq s \leq 4} \lambda_{F}^{c, r}(s) .
$$

When $s^{*}$ does not exist, we can immediately get that

$$
\min _{0 \leq s \leq 4} \lambda_{F}^{a}(s)=\min \left\{\lambda_{F}^{a}(0), \lambda_{F}^{a}(4)\right\}=\lambda_{F}^{a}(4)<\min _{0 \leq s \leq 4} \lambda_{F}^{c, r}(s) .
$$

We now combine this result with (5.7) and summarize the stability relation between the fully atomistic model and the reconstruction-based local model by the following theorem. 
Theorem 5.2. The relation between the stability of the reconstruction-based local model and the atomistic model depends on the sign of $\phi_{2 F}^{\prime \prime}+G_{F}^{\prime} \cdot 2 \rho_{2 F}^{\prime \prime}$ and is given by

$$
\begin{aligned}
& \min _{0 \leq s \leq 4} \lambda_{F}^{a}(s)=\lambda_{F}^{a}(4)<\min _{0 \leq s \leq 4} \lambda_{F}^{c, r}(s) \quad \text { if } \quad \phi_{2 F}^{\prime \prime}+G_{F}^{\prime} \cdot 2 \rho_{2 F}^{\prime \prime}>0, \\
& \min _{0 \leq s \leq 4} \lambda_{F}^{a}(s)=\min \left\{\lambda_{F}^{a}(0), \lambda_{F}^{a}\left(s^{*}\right), \lambda_{F}^{a}(4)\right\}=\min _{0 \leq s \leq 4} \lambda_{F}^{c, r}(s) \quad \text { if } \quad \phi_{2 F}^{\prime \prime}+G_{F}^{\prime} \cdot 2 \rho_{2 F}^{\prime \prime}=0, \\
& \min _{0 \leq s \leq 4} \lambda_{F}^{a}(s)=\min \left\{\lambda_{F}^{a}(0), \lambda_{F}^{a}\left(s^{*}\right), \lambda_{F}^{a}(4)\right\}>\min _{0 \leq s \leq 4} \lambda_{F}^{c, r}(s) \quad \text { if } \quad \phi_{2 F}^{\prime \prime}+G_{F}^{\prime} \cdot 2 \rho_{2 F}^{\prime \prime}<0 .
\end{aligned}
$$

We note from the theorem that the reconstruction-based local model can be less stable than the fully atomistic model, which might cause stability problems when constructing a coupling method.

\section{Conclusion.}

In this paper, we give precise estimates for the lattice stability of atomistic chains modeled by the fully atomistic EAM model and the volume-based and the reconstruction-based local approximations. We identify the critical assumptions for the pair potential, the electron density function, and the embedding function to study lattice stability. We find that both the volume-based local model and the reconstruction-based local model can give $\mathrm{O}(1)$ errors for the critical strain. The critical strain predicted by the volume-based model is always larger than that predicted by the atomistic model, but the critical strain for reconstruction-based models can be either larger or smaller than that predicted by the atomistic model.

Further research is needed to determine the significance of these results for multidimensional lattice stability and for atomistic-to-continuum coupling methods that couple an atomistic region with a volume-based local region through a reconstruction-based local region [5, 18].

\section{REFERENCES}

[1] X. Blanc, C. Le Bris, and F. Legoll. Analysis of a prototypical multiscale method coupling atomistic and continuum mechanics. M2AN Math. Model. Numer. Anal., 39(4):797-826, 2005.

[2] W. Curtin and R. Miller. Atomistic/continuum coupling in computational materials science. Modell. Simul. Mater. Sci. Eng., 11(3):R33-R68, 2003.

[3] M. Dobson, M. Luskin, and C. Ortner. Accuracy of quasicontinuum approximations near instabilities. Journal of the Mechanics and Physics of Solids, 58:1741-1757, 2010. arXiv:0905.2914v2.

[4] M. Dobson, M. Luskin, and C. Ortner. Sharp stability estimates for force-based quasicontinuum methods. SIAM J. Multiscale Modeling \& Simulation, 8:782-802, 2010. arXiv:0907.3861.

[5] W. E, J. Lu, and J. Yang. Uniform accuracy of the quasicontinuum method. Phys. Rev. B, 74(21):214115, 2006.

[6] W. E and P. Ming. Cauchy-Born rule and the stability of crystalline solids: static problems. Arch. Ration. Mech. Anal., 183(2):241-297, 2007.

[7] S. M. Foiles, M. I. Baskes, and M. S. Daw. Embedded-atom-method functions for the FCC metals Cu, Ag, Au, Ni, Pd, P, and their alloys. Phys. Rev. B, 33:7983-7911, 1986.

[8] T. Hudson and C. Ortner. On the stability of Bravais lattices and their Cauchy-Born approximations. ESAIM:M2AN, 46:81-110, 2012.

[9] R. A. Johnson and D. J. Oh. Analytic embedded atom method model for BCC metals. JMR., 4:1195-1201, 1989.

[10] X. H. Li and M. Luskin. An analysis of the quasi-nonlocal quasicontinuum approximation of the embedded atom model. International Journal for Multiscale Computational Engineering, to appear. arXiv:1008.3628v4.

[11] P. Lin. Convergence analysis of a quasi-continuum approximation for a two-dimensional material without defects. SIAM J. Numer. Anal., 45(1):313-332 (electronic), 2007.

[12] R. Miller and E. Tadmor. The quasicontinuum method: overview, applications and current directions. Journal of Computer-Aided Materials Design, 9:203-239, 2003.

[13] R. Miller and E. Tadmor. Benchmarking multiscale methods. Modelling and Simulation in Materials Science and Engineering, 17:053001 (51pp), 2009.

[14] Y. Mishin, M. J. Mehl, D. A. Papaconstantopoulos, A. F. Voter, and J. D. Kress. Structural stability and lattice defects in copper: Ab initio, tight-binding, and embedded-atom calculations. Phys. Rev. B, 63, 2001. 
[15] M. Ortiz, R. Phillips, and E. B. Tadmor. Quasicontinuum analysis of defects in solids. Philosophical Magazine A, 73(6):1529-1563, 1996 .

[16] A. V. Shapeev. Consistent energy-based atomistic/continuum coupling for two-body potential: 1D and 2D case. Multiscale Modeling and Simulation, to appear. arXiv:1010.0512

[17] V. B. Shenoy, R. Miller, E. B. Tadmor, D. Rodney, R. Phillips, and M. Ortiz. An adaptive finite element approach to atomic-scale mechanics-the quasicontinuum method. J. Mech. Phys. Solids, 47(3):611-642, 1999.

[18] T. Shimokawa, J. Mortensen, J. Schiotz, and K. Jacobsen. Matching conditions in the quasicontinuum method: Removal of the error introduced at the interface between the coarse-grained and fully atomistic region. Phys. Rev. B, 69(21):214104, 2004.

[19] B. Van Koten, X. H. Li, M. Luskin, and C. Ortner. A computational and theoretical investigation of the accuracy of quasicontinuum methods. In I. Graham, T. Hou, O. Lakkis, and R. Scheichl, editors, Numerical Analysis of Multiscale Problems. Springer, to appear. arXiv:1012.6031.

[20] B. Van Koten and M. Luskin. Analysis of energy-based blended quasicontinuum approximations. SIAM. J. Numer. Anal., to appear. arXiv:1008.2138. 